\title{
Design and Evaluation of Digital Baseband Converter Sub-channel Delay Compensation Method on Bandwidth Synthesis
}

\author{
Kun JIANG ${ }^{1}$, Pingbo $Y A N^{2}$, Yuanqin $W A N G^{2}$, Yiwen JIAO ${ }^{2}$, Xin $L I A N^{2}$, Ke XU \\ ${ }^{1}$ Beijing Inst. of Tracking and Telecommunication Technology, Beijing 100094, China \\ ${ }^{2}$ Academy of Equipment, Beijing 101416, China \\ ${ }^{3}$ China Xi'an Satellite Control Center, Shanxi Xi'an 710043, China \\ jiangkunzzy@126.com
}

\begin{abstract}
The effect of sub-channel delay on bandwidth synthesis is investigated to eliminate the "phase step" phenomenon in bandwidth synthesis during the test of CDBE (Chinese Digital Backend). Through formula derivation, we realize that sub-channel delay may cause phase discontinuity between different sub-channels. Theoretical analysis shows that sub-channel delay can induce bandwidth synthesis error in group delay measurement of the linear system. Furthermore, in the differential delay measurement between two stations, bandwidth synthesis error may occur when the LO (Local Oscillator) frequency differences of corresponding sub-channels are not identical. Error-free conditions are discussed under different applications. The phase errors among different sub-channels can be removed manually. However, the most effective way is the compensation of sub-channel delay. A sub-channel delay calculation method based on Modelsim is proposed. The compensation method is detailed. Simulation and field experiments are presented to verify our approach.
\end{abstract}

\section{Keywords}

VLBI, bandwidth synthesis, digital baseband converter, phase step, delay compensation, subchannel delay

\section{Introduction}

VLBI (Very Long Baseline Interferometry) has become a significant approach in astronomy, geodesy and deep space exploration due to its long operation range and extremely high angular resolution. As the requirement of measurement precision is increasing dramatically, IVS (International VLBI Service for Geodesy \& Astrometry) has launched VLBI2010 project to achieve 1-mm position accuracy in the future [1]. A new generation VLBI system has been built to overcome the limitations of the existing infrastructures. As the emergence of Digital Backend/ Digital Baseband Converter, more attentions have been paid to the issues on high sampling rate, better in-band performance, high flexibility and efficient implementation methods [2-4]. Little attention has been paid to the subchannel delay. Typical systems include RDBE [5] by NRAO (National Radio Astronomy Observatory) and MIT Haystack, the most recent DBBC3 [6] in EVN (European VLBI Network), ADS3000+ [7], CDAS (Chinese Data Acquisition System) [8] and CDBE (Chinese Digital Backend) [9].

Rogers [10] proposed bandwidth synthesis theory first in 1970 and discussed the factors that determine the optimal choice of frequency channels. Then, Molinder [11] detailed the tradeoff between bandwidth synthesis measurement accuracy and spanned bandwidth, source strength, antenna size and efficiency, system noise temperature, and data volume. Further, Gorham [12] proposed an approach toward choosing optimal sets of frequency channel for better bandwidth synthesis measurement accuracy. Bagri [13] introduced an accurate spacecraft measurement method based on frequency synthesis and the error budget was explained in detail. Recently, the influence of clockoffsets differences among different channels on bandwidth synthesis were investigated in [14]. A method based on a priori delay, whose derivatives up to the third order, was proposed to eliminate the influence. However, little research has been conducted on the impact of digital baseband converter's sub-channel delay on bandwidth synthesis.

We designed and implemented a novel VLBI Digital Baseband converting and recording system named CDBE (Chinese Digital Backend) in 2012 [9]. "Phase step" phenomenon was observed in the measurement of CDBE's single station group delay, and differential delay between two CDBEs by bandwidth synthesis. Therefore, in this paper, we first analyze the influence of digital baseband converter's sub-channel delay on bandwidth synthesis, and two conclusions are reached 1) Sub-channel delay will deteriorate bandwidth synthesis accuracy in the group delay measurement of a linear system; 2) Sub-channel delay will also affect the measurement accuracy of differential delay between two stations when the corresponding 
LO frequency differences are not identical. Based on the analysis, we believe that the most effective way to eliminate the influence of sub-channel delay is to compensate it to zero. Thus, we proposed an efficient dynamic sub-channel delay measurement method based on Modelsim. Furthermore, the sub-channel delay compensation strategy is presented on FPGA. Simulation results show that bandwidth synthesis accuracy was improved by two or three orders of magnitude with delay compensation. Field experiments based on CDBE also demonstrate the validity of our proposed compensation method.

The paper is organized as follows. In Sec. 2, reasons of "phase step" are derived respectively in the measurement of single station's group delay and differential delay between two stations. Next, influencing factors are discussed for different digital baseband converter implementation structures and an effective way to eliminate "phase step" is proposed. Section 3 describes how to calculate sub-channel delay and the compensation method is presented in detail. Simulations and field experiments based on CDBE are performed to verify the effect of sub-channel compensation in Sec. 4 and Sec. 5. Finally, conclusions are summarized in Sec. 6.

\section{Influence of Sub-channel Delay on Bandwidth Synthesis}

The VLBI delay measurement precision is proportional to the recording bandwidth. The wider the recording bandwidth, the higher is the measurement accuracy. However, the recording bandwidth is limited by the recording speed. It's impossible to record signal with ultra wide bandwidth. To overcome this limitation, bandwidth synthesis [10] was proposed by Rogers to obtain a comparative high measurement accuracy by limiting the total bandwidth. According to bandwidth synthesis theory, wideband signal is divided into many separate narrow band sub-channels in the frequency domain. The delay and delay rate of each sub-channel is obtained at first. Then, a more accurate delay can be obtained by a linear least square fitting to the measurement results of all the sub-channels. Bandwidth synthesis was initially proposed for the measurement of differential delay between two stations. It indicates a basic principle in delay measurement that the wider the measurement bandwidth, the more accurate is the delay measurement [15]. Therefore, the concept of bandwidth synthesis can be extended to the measurement of group delay of single station to improve its measurement precision.

\subsection{Group Delay Measurement of Single Station}

The RF (Radio Frequency) receiver illustrated in Fig. 1(a) is equivalent to the mathematical model in Fig. 1(b). For simplicity, assume that the RF receiver is a linear system, and the received signal is

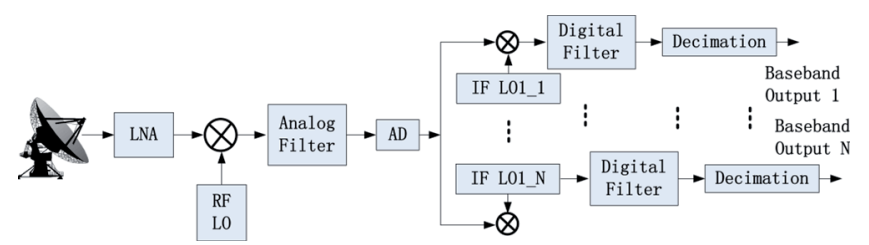

(a) Schematic of RF receiver

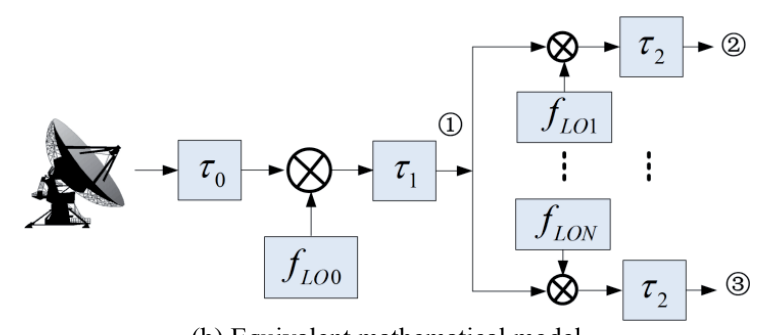

(b) Equivalent mathematical model

Fig. 1. RF receiver and the equivalent mathematical model.

$x(t)=\cos \left(2 \pi f_{1} t\right)+\cos \left(2 \pi f_{2} t\right)+\cos \left(2 \pi f_{3} t\right)+\cos \left(2 \pi f_{4} t\right)$

where $f_{1}, f_{2}, f_{3}$, and $f_{4}$ are the frequencies of the received tones. The phases of tones at label (1) (see Fig. 1(b)) are

$$
\left\{\begin{array}{l}
\Phi_{1_{-} 1}=-2 \pi f_{1} \tau_{0}-2 \pi\left(f_{1}-f_{L O 0}\right) \tau_{1} \\
\Phi_{1_{-} 2}=-2 \pi f_{2} \tau_{0}-2 \pi\left(f_{2}-f_{L O 0}\right) \tau_{1} \\
\Phi_{1_{-} 3}=-2 \pi f_{3} \tau_{0}-2 \pi\left(f_{3}-f_{L O 0}\right) \tau_{1} \\
\Phi_{1_{-} 4}=-2 \pi f_{4} \tau_{0}-2 \pi\left(f_{4}-f_{L O 0}\right) \tau_{1}
\end{array}\right.
$$

where $\tau_{0}$ is the delay before analog down-converting, $f_{L O 0}$ is the frequency of analog LO, $\tau_{1}$ is the delay before subchannel dividing. In (2), tones $f_{1}$ and $f_{2}$ are outputs from sub-channel 1 , while tones $f_{3}$ and $f_{4}$ are outputs from subchannel 2. Then, the tones' phases at label (2) and (3) (see Fig. 1(b)) are

$\left\{\begin{array}{l}\Phi_{2_{-} 1}=-2 \pi f_{1} \tau_{0}-2 \pi\left(f_{1}-f_{L O O}\right) \tau_{1}-2 \pi\left(f_{1}-f_{L O 0}-f_{L O 1}\right) \tau_{2} \\ \Phi_{2_{-} 2}=-2 \pi f_{2} \tau_{0}-2 \pi\left(f_{2}-f_{L O 0}\right) \tau_{1}-2 \pi\left(f_{2}-f_{L O 0}-f_{L O 1}\right) \tau_{2} \\ \Phi_{3_{-} 1}=-2 \pi f_{3} \tau_{0}-2 \pi\left(f_{3}-f_{L O 0}\right) \tau_{1}-2 \pi\left(f_{3}-f_{L O 0}-f_{L O N}\right) \tau_{2} \\ \Phi_{3_{-} 2}=-2 \pi f_{4} \tau_{0}-2 \pi\left(f_{4}-f_{L O 0}\right) \tau_{1}-2 \pi\left(f_{4}-f_{L O 0}-f_{L O N}\right) \tau_{2}\end{array}\right.$

Group delays derived by the tones in the same subchannel are

$$
\left\{\begin{array}{l}
\tau_{g d 1}=-\frac{\Phi_{2 \_2}-\Phi_{2 \_1}}{2 \pi\left(f_{2}-f_{1}\right)}=\tau_{0}+\tau_{1}+\tau_{2} \\
\tau_{g d N}=-\frac{\Phi_{3 \_2}-\Phi_{3 \_1}}{2 \pi\left(f_{4}-f_{3}\right)}=\tau_{0}+\tau_{1}+\tau_{2}
\end{array}\right.
$$

Equation (4) indicates that group delays of different sub-channels are identical. Therefore, the delay can be calculated by the tones in each sub-channel. For a linear system, the bandwidth synthesis group delay can be calculated by the two most outside tones $f_{1}$ and $f_{4}$ as in (5)

$$
\tau_{g d}=-\frac{\Phi_{3 \_}-\Phi_{2 \_1}}{2 \pi\left(f_{4}-f_{1}\right)}=\tau_{0}+\tau_{1}+\tau_{2}+\frac{f_{L O 1}-f_{L O N}}{f_{4}-f_{1}} \tau_{2} .
$$


By comparing (5) with (4), we find that there is an error term for the group delay calculated by different sub-channels' tones. The error term is determined by the sub-channel delay $\tau_{2}$, with a weighting factor $k$ as

$$
k=\frac{f_{L O 1}-f_{L O N}}{f_{4}-f_{1}} .
$$

The underlying reason for the occurrence of the error term in (5) is that different sub-channel LO frequencies break the original frequency relationship before demux. When the tones of different sub-channels pass the same delay, the corresponding phase variances are different from the original ones. Thus, a phase error $\Delta \varphi_{i}$ is added, as shown in Fig. 2.

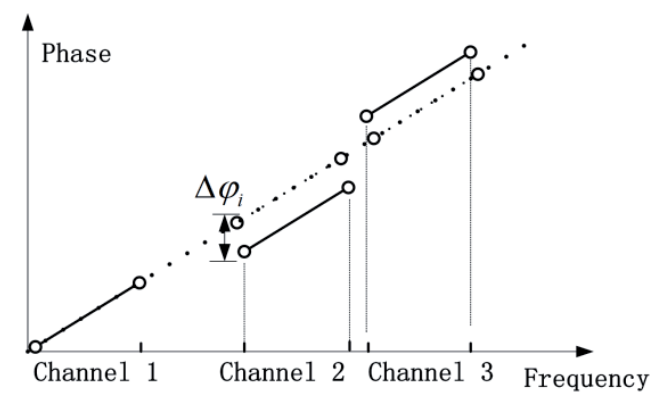

Fig. 2. Sketch of the "phase step" phenomenon in bandwidth synthesis.

In Fig. 2, the dotted line denotes the tones' nominal phases. The solid lines denote the actual phases of the tones in different sub-channels. We can see that the group delay of each sub-channel is identical (the slopes are the same). However, the actual phases are located around the nominal line. So we cannot obtain the delay correctly by bandwidth synthesis. We call this phenomenon as "phase step". The above conclusion is derived from absolute phase values of tones. In practice, although the phases may be ambiguous, our conclusion still fits.

The phase errors among different sub-channels can be eliminated manually by calculating the specific error values. However, the calculation is tedious, inefficient, and of low accuracy. From (5), we can derive a conclusion that if and only if $f_{L O 1}=f_{L O N}$ or $\tau_{2}=0$, the error is zero. Whereas, the LO frequency of each sub-channel is arbitrary. The condition $f_{L O 1}=f_{L O N}$ is not always satisfied. Furthermore, LO frequencies are not equal in the application of bandwidth synthesis. Therefore, we draw a conclusion that the sub-channel delay has to be compensated to zero to eliminate the error term.

\subsection{Differential Delay Measurement between Two Stations}

Assume the differential delay between station 2 and station 1 is $\tau_{\mathrm{g}}$. From (3), we can calculate the phases of the tones at station 2 as
$\left\{\begin{array}{l}\Phi_{2_{-} 1}^{\prime}=-2 \pi f_{1}\left(\tau_{0}+\tau_{g}\right)-2 \pi\left(f_{1}-f_{L O O}\right) \tau_{1}-2 \pi\left(f_{1}-f_{L O O}-f_{L O 1}^{\prime}\right) \tau_{2} \\ \Phi_{2 \_}^{\prime}=-2 \pi f_{2}\left(\tau_{0}+\tau_{g}\right)-2 \pi\left(f_{2}-f_{L O 0}\right) \tau_{1}-2 \pi\left(f_{2}-f_{L O 0}-f_{L O 1}^{\prime}\right) \tau_{2} \\ \Phi_{3 \_1}^{\prime}=-2 \pi f_{3}\left(\tau_{0}+\tau_{g}\right)-2 \pi\left(f_{3}-f_{L O 0}\right) \tau_{1}-2 \pi\left(f_{3}-f_{L O 0}-f_{L O N}^{\prime}\right) \tau_{2} \\ \Phi_{3_{-} 2}^{\prime}=-2 \pi f_{4}\left(\tau_{0}+\tau_{g}\right)-2 \pi\left(f_{4}-f_{L O 0}\right) \tau_{1}-2 \pi\left(f_{4}-f_{L O 0}-f_{L O N}^{\prime}\right) \tau_{2}\end{array}\right.$

According to the correlation of the tones between two stations, the differential phases are

$$
\left\{\begin{array}{l}
\Delta \Phi_{2_{-} 1}=\Phi_{2_{-} 1}-\Phi_{2_{-} 1}^{\prime}=2 \pi f_{1} \tau_{g}+2 \pi\left(f_{L O 1}-f_{L O 1}^{\prime}\right) \tau_{2} \\
\Delta \Phi_{2_{-} 2}=\Phi_{2_{-} 2}-\Phi_{2_{-} 2}^{\prime}=2 \pi f_{2} \tau_{g}+2 \pi\left(f_{L O 1}-f_{L O 1}^{\prime}\right) \tau_{2} \\
\Delta \Phi_{3_{-} 1}=\Phi_{3_{-} 1}-\Phi_{3_{-1} 1}^{\prime}=2 \pi f_{3} \tau_{g}+2 \pi\left(f_{L O N}-f_{L O N}^{\prime}\right) \tau_{2} \\
\Delta \Phi_{3{ }_{3} 2}=\Phi_{3_{-} 2}-\Phi_{3_{-} 2}^{\prime}=2 \pi f_{4} \tau_{g}+2 \pi\left(f_{L O N}-f_{L O N}^{\prime}\right) \tau_{2}
\end{array}\right.
$$

Then, differential delays between two stations can be obtained by the tones in the corresponding sub-channels

$$
\left\{\begin{array}{l}
\tau_{g 1}=-\frac{\Delta \Phi_{2 \_2}-\Delta \Phi_{2 \_1}}{2 \pi\left(f_{2}-f_{1}\right)}=\tau_{g} \\
\tau_{g N}=-\frac{\Delta \Phi_{3 \_2}-\Delta \Phi_{3 \_1}}{2 \pi\left(f_{4}-f_{3}\right)}=\tau_{g}
\end{array}\right.
$$

From (9), we can see that the differential delays calculated by corresponding sub-channels are the same and correct. Then, according to bandwidth synthesis theory, we can obtain the differential delay by a wider bandwidth, tones $f_{1}$ and $f_{4}$ as

$$
\begin{aligned}
\tau_{g}^{\prime} & =-\frac{\Delta \Phi_{3 \_2}-\Delta \Phi_{2 \_1}}{2 \pi\left(f_{4}-f_{1}\right)} \\
& =\tau_{g}+\frac{\left(f_{L O N}-f_{L O N}^{\prime}\right)-\left(f_{L O 1}-f_{L O 1}^{\prime}\right)}{f_{4}-f_{1}} \tau_{2} \\
& =\tau_{g}+\Delta \tau_{g}
\end{aligned}
$$

where $\Delta \tau_{g}$ is the error term, $\Delta f_{N}=f_{L O N}-f_{L O N}^{\prime}$, and $\Delta f_{1}=f_{L O 1}-f_{L O 1}^{\prime}$. From (10), we can derive that when one of the following three conditions is satisfied, the error term is zero:
a) $f_{L O N}=f_{L O N}^{\prime}$ and $f_{L O 1}=f_{L O 1}^{\prime}$;
b) $\Delta f_{N}=\Delta f_{1}$;
c) $\tau_{2}=0$.

\subsubsection{The LO Frequency is Equal to Each Other}

Condition (a) is the special case of condition (b). Assume the tone emitted by the radio source or spacecraft is $f$. Then, the received tones at two observation stations become $f^{\prime}$ and $f^{\prime}+f_{d}$, respectively, where $f_{d}$ is the Doppler frequency difference between two stations.

If $f_{d}<B$, where $B$ is the recording bandwidth, the corresponding LO frequencies can be set to be the same, and condition (a) is satisfied. 
Whereas, if $f_{d}>B$, the corresponding LO frequencies should be different. Otherwise, the received tones at one of the stations may be located outside the recording bandwidth. Here, condition (a) cannot be satisfied.

Condition (a) can be satisfied in the observation of the radio source. The received signal is a kind of wideband signal. Thus, the recording bandwidth is usually wider than Doppler frequency difference.

Whereas, for the observation of spacecraft, the received signals are usually tones, and the recording bandwidth is relatively narrow. Therefore, condition (a) may not be satisfied in some cases.

\subsubsection{The Difference of Corresponding LO Frequency is Equal to Each Other}

Condition (b) is related to the down-conversion scheme of digital baseband converter. Assume the tones received at station 1 are $f_{s 1}$ and $f_{s 1}+\Delta f$, where $\Delta f$ is the frequency difference between the tones. Tone $f_{s 1}$ passes through sub-channel 1 , and Tone $f_{s 1}+\Delta f$ passes through sub-channel 2. Correspondingly, the tones received at station 2 are $f_{s 1}+f_{d}$ and $f_{s 1}+f_{d}+\Delta f$, where $f_{d}$ is the Doppler frequency difference between two stations. Tone $f_{s 1}+f_{d}$ passes through sub-channel 1 , and Tone $f_{s 1}+f_{d}+\Delta f$ passes through sub-channel 2. Both of the tones at the two stations are down-converted to zero. Then the condition is investigated according to different down-conversion schemes of digital baseband converter.

Firstly, for the digital baseband converter with the orthogonal mixing scheme, the frequency relationship between tones does not change after down-conversion. So the LO frequencies of different sub-channels have the relationship as

$$
\begin{aligned}
& f_{s 1 L O 2}=f_{s 1 L O 1}+\Delta f, \\
& f_{s 2 L O 2}=f_{s 2 L O 1}+\Delta f
\end{aligned}
$$

where $f_{s 1 L O 1}$ and $f_{s 1 L O 2}$ are the LO frequencies of subchannel 1 and 2 at station $1, f_{s 2 \mathrm{LO} 1}$ and $f_{s 2 \mathrm{LO} 2}$ are the LO frequencies of sub-channel 1 and 2 at station 2. From (11) and (12), the following equation can be obtained

$$
f_{s 2 L O 2}-f_{s 1 L O 2}=f_{s 2 L O 1}-f_{s 1 L O 1} .
$$

Consequently, condition (b) is satisfied.

Secondly, for the digital baseband converter with the PFB (Polyphase Filter Bank)+FFT scheme, once the channelization structure is confirmed, the LO frequency of each sub-channel is constant. If the two stations have the same channelization structure, the corresponding LO frequencies are the same. Then, condition (a) is satisfied.

Thirdly, for the digital baseband converter with the combined structure of PFB+FFT and orthogonal mixing scheme, such as CDBE, the cases are complicated. The channelization structure has broken the original frequency relationship. So condition (b) is not always satisfied.

\subsubsection{The Sub-channel Delay is Zero}

In the actual observation, the digital baseband converters at the two stations may be different in down-conversion schemes, so the cases may be very complicated [16], [17]. Condition (a) and (b) both have limitations. Therefore, the most simple, general, and reliable approach is to compensate the sub-channel delay to zero.

\section{Calculation and Compensation of Sub-channel Delay}

\subsection{Sub-channel Delay Calculation Methods}

The process of down-converting is implemented digitally on FPGA. The delay of a digital circuit is constant. So once the scheme of digital baseband converter is confirmed, the delay of each module in a sub-channel can be calculated separately. The delay summation of all the modules is the sub-channel delay.

The main module, which delays the signal, is a digital filter. VLBI is a strict linear system. The digital filters adopted are all FIR filters. The group delay of FIR filter is related to its architecture. Different architecture has different group delay. For the common symmetric distributed architecture, the relationship between the group delay and the number of filter coefficients is

$$
\tau_{\text {filter }}=\frac{N-1}{2} T_{\text {clock }}
$$

where $N$ is the number of filter coefficients, $T_{\text {clock }}$ is the working clock cycle.

Static method is tedious and requires high professional knowledge. Filter delay of each stage should be calculated. In addition, the specific architecture of each filter should be known clearly.

To realize the easy, efficient and reliable measurement of sub-channel delay, a novel dynamic measuring method is proposed. In dynamic method, the sub-channel module is treated as a black box. Sub-channel delay is measured by Modelsim simulation. The detailed processing flow is shown in Fig. 3.

First of all, testing signal is generated in Matlab. Digital PCAL (Phase Calibration) signal is adopted in this step. Then, related VHDL/Verilog files are loaded into Modelsim project. Write the testbench according to the input/out put interface and timing relationships. Make a simulation,

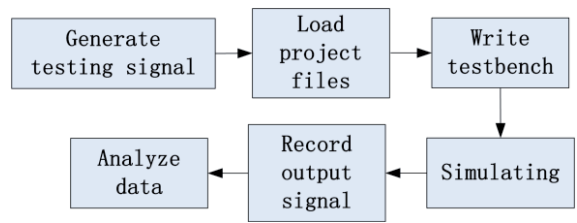

Fig. 3. Flow chart of dynamic measurement method. 
and record the outputs meanwhile. Afterwards, calculate the phases of PCAL tones in Matlab [18]. Finally, the group delay of sub-channel is obtained.

Dynamic method does not require knowledge of the architecture of the filter. It is efficient to obtain the subchannel delay. The result is reliable since it is simulating actual cases.

\subsection{Sub-channel Delay Compensation Method}

In design and implementation of our proposed compensation method, we adopt a data stream based processing strategy. Each data stream is attached with a valid signal to control its validity, which makes the delay compensation easy. We should only obtain the sub-channel delay by dynamic method first, and then convert the delay to working clock cycles. Finally, we shift the data valid signal according to the clock cycles, and then the sub-channel delay is compensated.
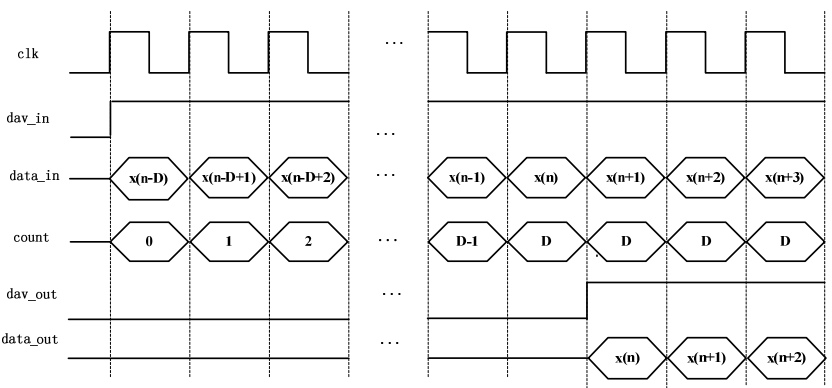

Fig. 4. Timing diagram of delay compensation module.

As illustrated in Fig. 4, clk is the working clock, dav_in is the input data valid signal, data in is the input signal, count represents the working clock cycle counter, dav_out is the output data valid signal, data_out is the output signal. Suppose the filter delay equals to D working clock cycles. Delay compensation is to drop the first D output signal data and delay the dav_out D working clock cycles. Then the Sub-channel delay is compensated to zero.

There are three advantages of sub-channel delay compensation.

1) Phases among different sub-channels can realize seamless combination directly by the compensation of subchannel delay. Thus, the bandwidth synthesis fitting precision can be improved.

2) The total delay of digital baseband converter is constant and independent of the output bandwidth, which makes the delay compensation in VLBI correlator easier.

3) The total delay of station instrument decreases by the compensation of sub-channel delay, which can diminish or eliminate the phase ambiguity of PCAL signals.

\section{Simulation}

\subsection{Group Delay Measurement of Single Station}

To verify the effectiveness of sub-channel delay compensation, CDBE models based on the combined structure of PFB+FFT and orthogonal mixing down-conversion were built [19]. Digital PCAL signal with a $2 \mathrm{MHz}$ PRF (Pulse Repetition Frequency) was generated in Matlab as the testing signal. The number of output sub-channels was

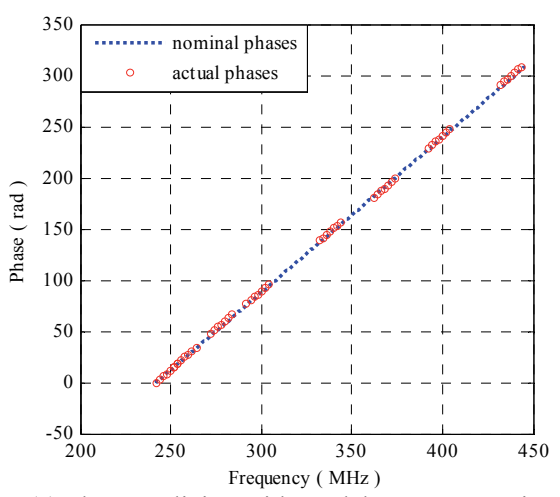

(a) Phases splicing without delay compensation.

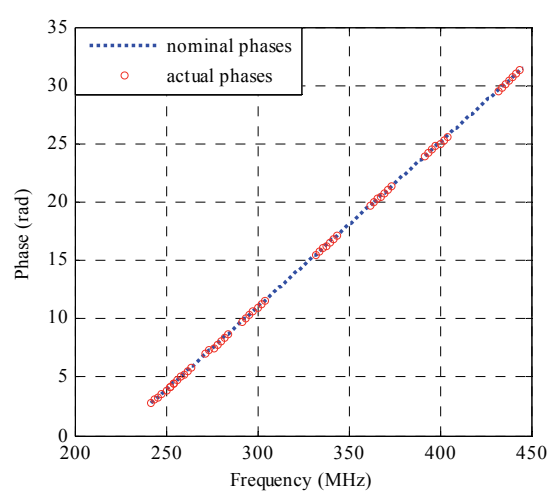

(b) Phases splicing with delay compensation.

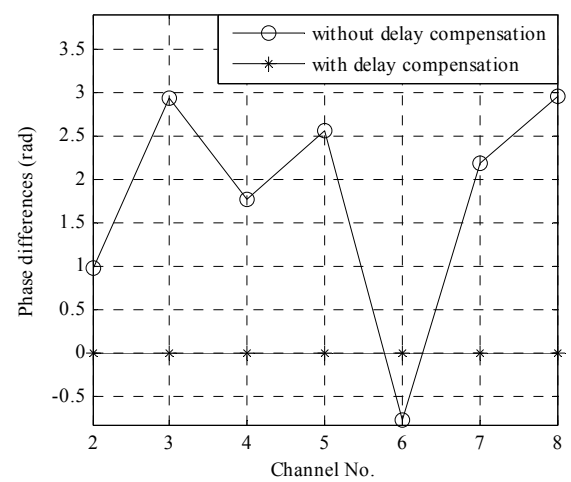

(c) Phase differences between sub-channels and nominal line.

Fig. 5. Phases splicing of different sub-channels. 
eight, and the output bandwidth was $16 \mathrm{MHz}$. The digital LO frequencies of sub-channels were set at $240 \mathrm{MHz}$, $250 \mathrm{MHz}, \quad 270 \mathrm{MHz}, 290 \mathrm{MHz}, \quad 330 \mathrm{MHz}, \quad 360 \mathrm{MHz}$, $390 \mathrm{MHz}$, and $430 \mathrm{MHz}$, respectively, with a bandwidth span of $206 \mathrm{MHz}$. CDBE models with/without sub-channel delay compensation were simulated separately in Modelsim. The simulation outputs were recorded, and then analyzed in Matlab. The tones' phases of each sub-channel were unwrapped and spliced together, as shown in Fig. 5.

The dotted line in Fig. 5(a), (b) denotes the nominal phase line, which is the extension of phases of sub-channel 1. The circled lines in Fig. 5(a), (b) denote the phases of the other sub-channels, which are supposed to fit the nominal line. In order to see the "phase step" clearly, we obtain the mean phase differences between the actual phases and the nominal ones of each channel, shown in Fig. 5(c). From Fig. 5(c), it is clear to see that the phase differences without sub-channel delay compensation are relatively large. The "phase step" is evident. In contrast, after delay compensation, the phase differences decrease to the order of $0.001 \mathrm{rad}$, which indicates that delay compensation is effective.

By the linear least square fitting of each sub-channel and the entire bandwidth, the group delays and RMS (Root Mean Square) errors of each sub-channel and bandwidth synthesis are shown in Tab. 1.

\begin{tabular}{|c|c|c|c|c|}
\hline \multirow{2}{*}{$\begin{array}{c}\text { Sub-Channel } \\
\text { Number }\end{array}$} & \multicolumn{2}{|c|}{$\begin{array}{c}\text { Without Delay } \\
\text { Compensation }\end{array}$} & \multicolumn{2}{c|}{$\begin{array}{c}\text { With Delay } \\
\text { Compensation }\end{array}$} \\
\cline { 2 - 5 } & $\begin{array}{c}\text { Delay } \\
\text { (ns) }\end{array}$ & $\begin{array}{c}\text { RMS } \\
\text { (ns) }\end{array}$ & $\begin{array}{c}\text { Delay } \\
(\text { ns })\end{array}$ & $\begin{array}{c}\text { RMS } \\
(\text { ns })\end{array}$ \\
\hline Channel 1 & 241.190 & 0.017 & 22.464 & 0.022 \\
\hline Channel 2 & 241.126 & 0.024 & 22.394 & 0.038 \\
\hline Channel 3 & 241.242 & 0.039 & 22.496 & 0.035 \\
\hline Channel 4 & 241.175 & 0.040 & 22.412 & 0.044 \\
\hline Channel 5 & 241.210 & 0.025 & 22.453 & 0.035 \\
\hline Channel 6 & 241.195 & 0.014 & 22.435 & 0.024 \\
\hline Channel 7 & 241.163 & 0.035 & 22.412 & 0.065 \\
\hline Channel 8 & 241.245 & 0.037 & 22.475 & 0.048 \\
\hline Bandwidth Synthesis & $\mathbf{2 4 2 . 7 3 1}$ & $\mathbf{0 . 8 8 4}$ & $\mathbf{2 2 . 4 6 2}$ & $\mathbf{0 . 0 0 2}$ \\
\hline
\end{tabular}

Tab. 1. Group delays and RMS errors without / with delay compensation.

Tab. 1 indicates that the delay of CDBE decreases greatly from $241 \mathrm{~ns}$ to $22 \mathrm{~ns}$ when sub-channel delay is compensated. The residual $22 \mathrm{~ns}$ is the common delay before divided into sub-channels. The accuracy of bandwidth synthesis increases by two orders of magnitude by compensating sub-channel delay from $0.884 \mathrm{~ns}$ to $0.002 \mathrm{~ns}$. Furthermore, compared with the RMS errors of sub-channels with delay compensation, the measurement precision is improved from the order of $0.01 \mathrm{rad}$ to $0.001 \mathrm{rad}$ by bandwidth synthesis.

\subsection{Differential Delay Measurement of Double Stations}

In order to verify the conclusion that "phase step" phenomenon occurs when the differences of corresponding LO frequencies are not identical. We set the LO frequen- cies of station 1 at $240 \mathrm{MHz}, 250 \mathrm{MHz}, 270 \mathrm{MHz}$, $290 \mathrm{MHz}, 330 \mathrm{MHz}, 360 \mathrm{MHz}, 390 \mathrm{MHz}$, and $430 \mathrm{MHz}$, respectively. In contrast, the LO frequencies of station 2 were set at $240.5 \mathrm{MHz}, 250 \mathrm{MHz}, 270.5 \mathrm{MHz}, 290 \mathrm{MHz}$, $330.5 \mathrm{MHz}, 360 \mathrm{MHz}, 390.5 \mathrm{MHz}$, and $430 \mathrm{MHz}$, respectively. The differences between the corresponding LO frequencies were $0.5 \mathrm{MHz}$ or $0 \mathrm{MHz}$. Assume the signal of station 2 was delayed by 4.8828 ns compared with station 1 . Digital PCAL was adopted as the testing signal with a $2 \mathrm{MHz}$ PRF. The bandwidth was $16 \mathrm{MHz}$. CDBE models with/without sub-channel delay compensation were simulated separately in Modelsim by PCAL signal and delayed PCAL signal. Outputs of two stations were correlated to obtain the differential delays between the corresponding sub-channels. The bandwidth synthesis results were obtained by linear least square fitting of phase differences of all the sub-channels. The measurement results are shown in Tab. 2 and Fig. 6.

We can see clearly from Fig. 6(a) and Tab. 2 that the "phase step" phenomenon is evident and the bandwidth synthesis result cannot be obtained correctly before delay compensation. The "phase step" phenomenon disappears after delay compensation, and the bandwidth synthesis measurement accuracy is improved by one order of magnitude compared with the sub-channels' results. Simulation results demonstrate the correctness of our conclusion.

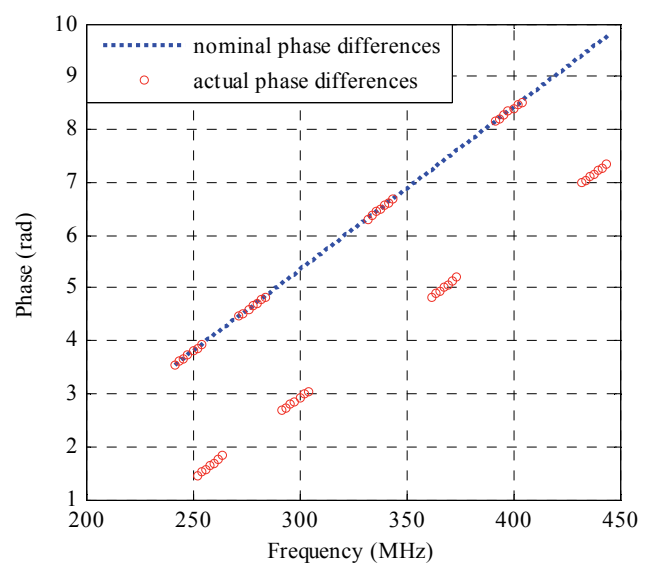

(a) Phase differences before delay compensation

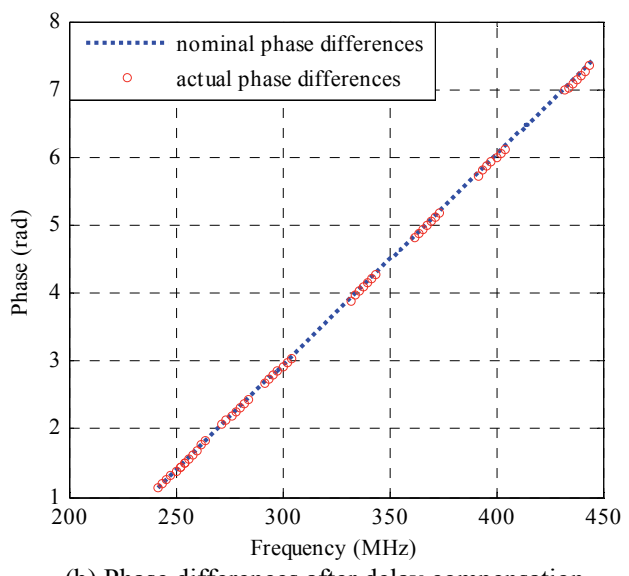

(b) Phase differences after delay compensation

Fig. 6. Phase differences with different LO frequency differences. 


\begin{tabular}{|c|c|c|c|c|}
\hline \multirow{2}{*}{$\begin{array}{c}\text { Sub-Channel } \\
\text { Number }\end{array}$} & \multicolumn{2}{|c|}{$\begin{array}{c}\text { Before Delay } \\
\text { Compensation }\end{array}$} & \multicolumn{2}{c|}{$\begin{array}{c}\text { After Delay } \\
\text { Compensation }\end{array}$} \\
\cline { 2 - 5 } & $\begin{array}{c}\text { Delay } \\
\text { (ns) }\end{array}$ & $\begin{array}{c}\text { RMS } \\
(\mathrm{ns})\end{array}$ & $\begin{array}{c}\text { Delay } \\
\text { (ns) }\end{array}$ & $\begin{array}{c}\text { RMS } \\
\text { (ns) }\end{array}$ \\
\hline Channel 1 & 4.886 & 0.020 & 4.913 & 0.018 \\
\hline CHANNEL 2 & 4.907 & 0.024 & 4.869 & 0.036 \\
\hline Channel 3 & 4.877 & 0.051 & 4.736 & 0.045 \\
\hline Channel 4 & 4.875 & 0.052 & 4.822 & 0.049 \\
\hline Channel 5 & 4.885 & 0.028 & 4.889 & 0.068 \\
\hline Channel 6 & 4.900 & 0.031 & 4.851 & 0.042 \\
\hline Channel 7 & 4.910 & 0.022 & 4.941 & 0.057 \\
\hline Channel 8 & 4.856 & 0.036 & 4.813 & 0.049 \\
\hline Bandwidth Synthesis & $\mathbf{4 . 1 4 9}$ & $\mathbf{1 . 3 1 0}$ & $\mathbf{4 . 8 8 2}$ & $\mathbf{0 . 0 0 3}$ \\
\hline
\end{tabular}

Tab. 2. Differential delays and RMS errors with different LO frequency differences.

\section{Field Experiments}

A series of field experiments were conducted to verify the effectiveness of sub-channel delay compensation in CDBE instrument.

\subsection{Phase Frequency Characteristic Measurement of Single Station}

A test system was established as shown in Fig. 7. The atomic clock was a Symmetricom Datum X72 rubidium clock, which generates $10 \mathrm{MHz}$ clock reference and $1 \mathrm{pps}$. The reference clock of PCAL generator and LO were at $100 \mathrm{MHz}$, which was synchronously generated by Agilent E8257C signal generator through the external $10 \mathrm{MHz}$ reference from the atomic clock. The whole system was coherent. PCAL generator can generate a comb signal with the bandwidth of $40 \mathrm{GHz}$, which was used as the testing signal.

Parameters of CDBE were set as follows. The number of output sub-channels was four. The output bandwidth was $16 \mathrm{MHz}$, with a quantization bit of 8 bit. The LO frequencies were set at $308 \mathrm{MHz}, 320 \mathrm{MHz}, 332 \mathrm{MHz}$, and $344 \mathrm{MHz}$, with an overlap of $4 \mathrm{MHz}$. Thus, the bandwidth span was $52 \mathrm{MHz}$. The PRF of comb signal was $100 \mathrm{kHz}$, and $S / \Phi$ was $30 \mathrm{dBHz}$.

PCAL signals were recorded and then analyzed to verify the delay compensation effectiveness. The phases of the tones in the overlap band of adjacent sub-channels are shown in Fig. 8.

The phases of the tones in the overlap bands coincide with each other, as shown in Fig. 8. Phase differences on both sides of the overlap bands are relatively large due to the effect of filter transition band. The average phase difference of tones in overlap band except both sides is $0.0019 \mathrm{rad}\left(0.109^{\circ}\right)$, which is equivalent to a group delay measurement RMS error of 8.22 ps for a linear system with a bandwidth of $52 \mathrm{MHz}$. The test results demonstrate that our proposed sub-channel delay compensation is effective, and the compensation precision is high.

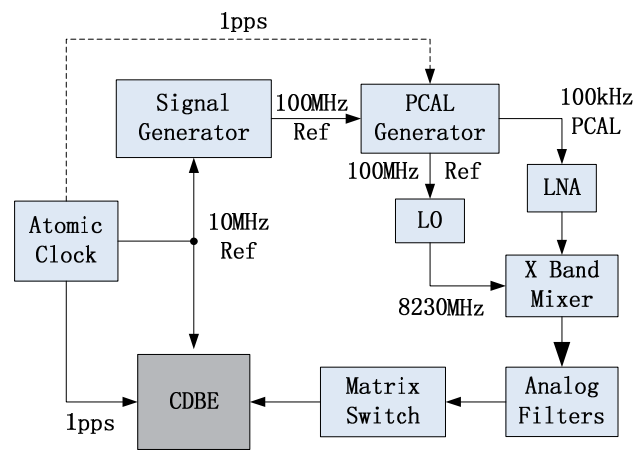

Fig. 7. Block diagram of single station test system.
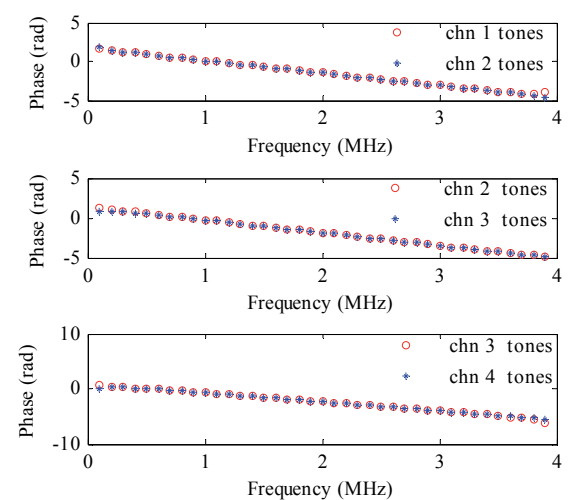

Fig. 8. Phases comparison of overlap tones in adjacent subchannels.

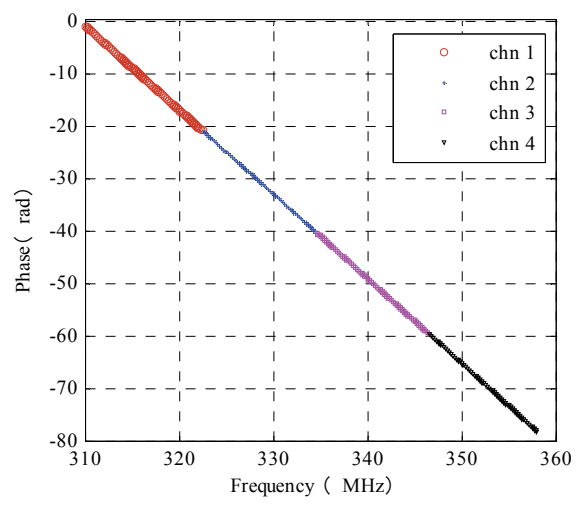

Fig. 9. Phase-frequency characteristics of the combination band.

The whole phase-frequency characteristic of the entire $52 \mathrm{MHz}$ bandwidth can be obtained by the phase combination of the sub-channels according to their original frequency relationship, which is illustrated in Fig. 9.

From Fig. 9, we can see that the phases change smoothly at the joints of adjacent channels, which verifies the validity of sub-channel delay compensation again. Due to the seamless combination of the phases of different subchannels, we can obtain the group delays of each subchannel and that of the entire band, as shown in Tab. 3 .

Tab. 3 demonstrates that group delays of the subchannels are different and the RMS error of bandwidth synthesis is larger than that of the sub-channels, which 
does not fit in with the expectation. The reason is that the analog transmission channel is nonlinear. The nonlinear characteristic of the testing band can be obtained by a linear polynomial fitting of phase-frequency characteristic, as shown in Fig .10.

\begin{tabular}{|c|c|c|}
\hline $\begin{array}{c}\text { Sub-Channel } \\
\text { Number }\end{array}$ & $\begin{array}{c}\text { Group Delay } \\
\text { (ns) }\end{array}$ & $\begin{array}{c}\text { RMS } \\
\text { (ns) }\end{array}$ \\
\hline Channel 1 & 255.283 & 0.149 \\
\hline Channel 2 & 254.783 & 0.251 \\
\hline Channel 3 & 257.615 & 0.211 \\
\hline Channel 4 & 258.786 & 0.102 \\
\hline Bandwidth Synthesis & $\mathbf{2 5 6 . 3 5 9}$ & $\mathbf{0 . 3 4 7}$ \\
\hline
\end{tabular}

Tab. 3. Linear group delays and RMS errors of the testing system.

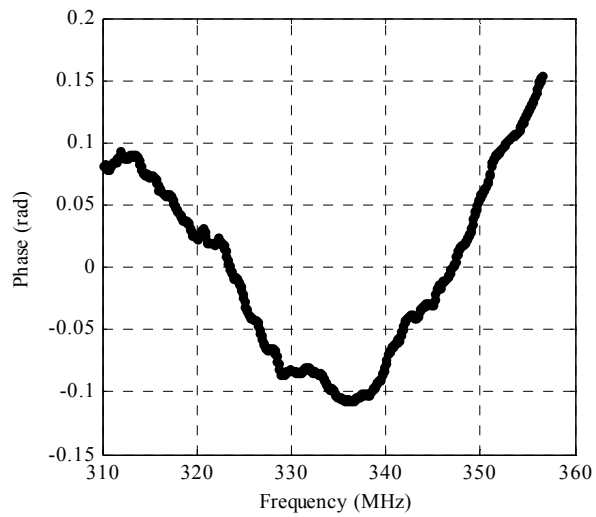

Fig. 10. Nonlinear characteristic of the whole test band.

\subsection{Zero Baseline Interferometry Test}

A ZBI (zero baseline interferometry) test was conducted to verify the effectiveness of our proposed subchannel delay compensation method in interferometry. The architecture of the testing system is shown in Fig. 11.

PCAL signal and channel noise were injected into LNA (Low Noise Amplifier) as the testing signal, which passed through the same path. The LO frequencies of each sub-channel of CDBE A were set at $270 \mathrm{MHz}, 282 \mathrm{MHz}$, $294 \mathrm{MHz}, 306 \mathrm{MHz}, 318 \mathrm{MHz}, 330 \mathrm{MHz}, 342 \mathrm{MHz}$, and $356 \mathrm{MHz}$, respectively. The LO frequencies of CDBE_B were set at $270 \mathrm{MHz}, 282.5 \mathrm{MHz}, 294 \mathrm{MHz}, 306.5 \mathrm{MHz}$,

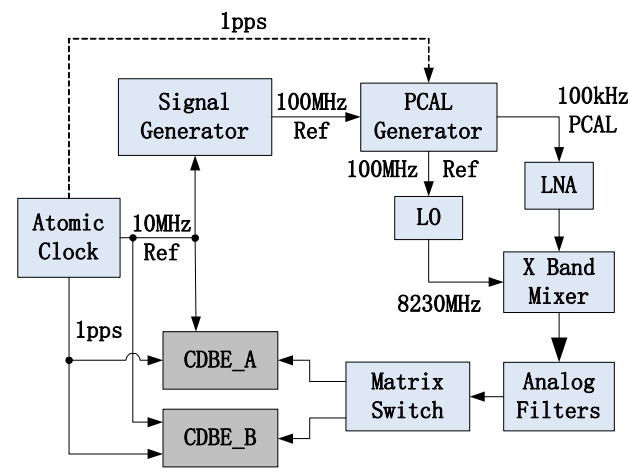

Fig. 11. Block diagram of zero baseband interferometry testing system.

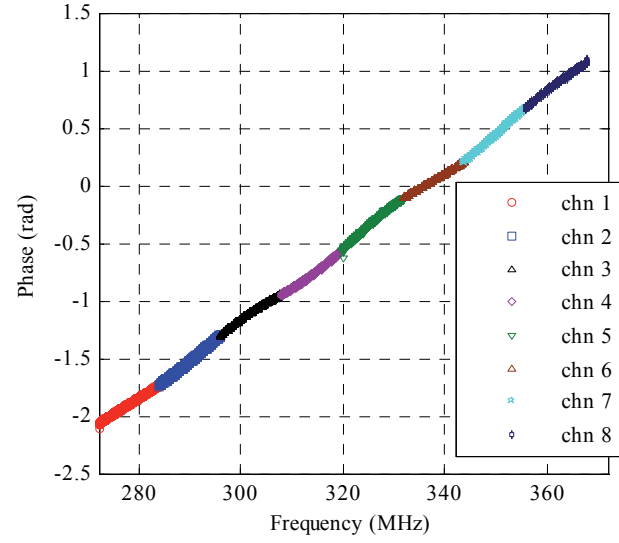

Fig. 12. Zero baseline interferometry results.

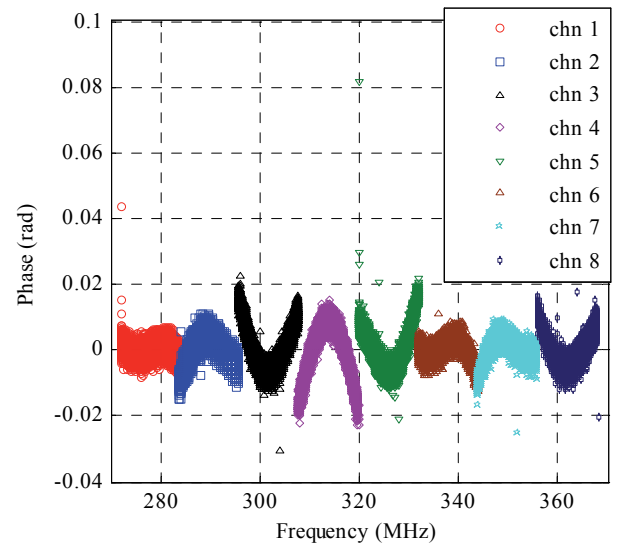

Fig. 13. Zero baseline interferometry results with PCAL calibration.

$318 \mathrm{MHz}, 330.5 \mathrm{MHz}, 342 \mathrm{MHz}$, and $356.5 \mathrm{MHz}$, respectively. The frequency differences of the corresponding LOs were $0.5 \mathrm{MHz}$ or $0 \mathrm{MHz}$, which were different. The other parameters were set the same as those in the single station test in Sec. 5.1.

The results of ZBI are presented in Fig. 12. We can see that the joints of adjacent sub-channels converge well, which indicates that our proposed sub-channel delay compensation is effective. Instrument differential delays between two CDBEs are involved in the ZBI results, which should be removed by PCAL signals. The phases calibrated by PCAL signals are illustrated in Fig. 13. The measurement results are shown in Tab. 4.

\begin{tabular}{|c|c|c|}
\hline $\begin{array}{c}\text { Sub-Channel } \\
\text { Number }\end{array}$ & $\begin{array}{c}\text { Differential Delay } \\
(\mathrm{ps})\end{array}$ & $\begin{array}{c}\text { RMS } \\
\text { (ns })\end{array}$ \\
\hline Channel 1 & 0.078 & 2.017 \\
\hline Channel 2 & 5.879 & 4.164 \\
\hline Channel 3 & 8.524 & 6.761 \\
\hline Channel 4 & 7.127 & 8.410 \\
\hline Channel 5 & 0.108 & 6.404 \\
\hline Channel 6 & 3.262 & 2.831 \\
\hline Channel 7 & 5.017 & 3.375 \\
\hline Channel 8 & 4.996 & 4.965 \\
\hline Bandwidth Synthesis & $\mathbf{0 . 0 8 6}$ & $\mathbf{0 . 5 9 0}$ \\
\hline
\end{tabular}

Tab. 4. Calibrated zero baseline interferometry results. 
From Fig. 13, we can see that the nonlinear characteristic of sub-channels is evident, which shows the differences of phase-frequency characteristic between the analog signal conditioning modules at the front of two CDBEs. Nonlinearity results in large RMS errors in sub-channel differential delay measurement, which can be improved by bandwidth synthesis as illustrated in Tab. 4 .

\section{Conclusion}

We realized that sub-channel delay of CDBE can introduce phase discontinuity between different sub-channels. For the group delay measurement of a linear system, the phase discontinuity will deteriorate the precision of bandwidth synthesis. For the measurement of differential delay between two stations, "phase step" phenomenon will occur when the corresponding LO frequency differences between the two stations are not identical. The phase errors among different sub-channels can be removed manually. However, the most effective way is to compensate for the sub-channel delay to zero.

A dynamic sub-channel delay measuring method based on Modelsim is proposed. Sub-channel delay can be compensated to zero by shifting calculated clock cycles of the attached data valid signal. Simulation results demonstrate that the accuracy of bandwidth synthesis can be improved by two or three orders of magnitude by our proposed sub-channel delay compensation method, when the corresponding LO frequency differences between the two stations are not identical. Field experiments results show that the sub-channel delay compensation is effective. The nonlinear characteristics of the whole test band can be obtained with sub-channel delay compensation.

The contribution of our proposed sub-channel delay compensation method can be summarized as follows:

1) Phases between different sub-channels can be combined seamlessly, which improves fitting accuracy of bandwidth synthesis.

2) The total delay of CDBE is constant and independent of output bandwidth, which makes the delay compensation in VLBI correlator easier.

3) The total delay of station instrument decreases, which can diminish or eliminate the phase ambiguity of PCAL signals.

\section{References}

[1] PETRACHENKO, B., NIELL, A., BEHREND, D., et al. Design aspects of the VLBI2010 system. Progress Report of the IVS VLBI2010 Committee. NASA/TM-2009-214180, 2009, p. 1-56.

[2] PETRACHENKO, B. VLBI2010 digital back end (DBE) requirements. IVS memorandum 2008-014v0, 2008, p. 1-7.

[3] COREY, B. Flexible down-converter. In VLBI2010 Workshop on Technical Specifications. Wettzell (Germany), 2012, p. 1-14.
[4] TUCCARI, G. Digital backends VLBI2010. In VLBI2010 Workshop on Technical Specifications. Wettzell (Germany), 2012, p. $1-25$.

[5] DURAND, S., HINTON, A. ET AL. Haystack/NRAO VLBI digital backend on the ROACH platform. In CASPR Workshop 2010. CASPR (USA), 2010, p. 1-14.

[6] TUCCARI, G., BUTTACCIO, S. DBBC3: VLBI at 32 Gbits per second. In 11th EVN Symp.\& Users Meeting. Bordeaux (France), 2012, p. 1-6.

[7] TAKEFUJ, K., TAKEUCHI, H., TSUTSUMI, M. Nextgeneration A/D sampler ADS3000+ for VLBI. In 2010 IVS General Meeting Proc. California (USA). NASA, 2010, p. 378 to 382.

[8] ZHU REN-JIE, ZHANG XIU-ZHONG, WEI WEN-REN, ET AL. The progress of modern Chinese data acquisition system. Progress in Astronomy, 2011, vol. 29, no. 2, p. 207-217.

[9] JIAO YIWEN, JIANG KUN, HOU XIAOMIN, ET AL. Design of the deep space interferometry digital backend. In SpaceOps 2012. Stockholm (Sweden), 2012, p. 1-6. DOI: 10.2514/6.2012-1342990

[10] ROGERS, A. E. E. Very long baseline interferometry with large effective bandwidth for phase-delay measurements. Radio Science, 1970, vol. 5, no. 10 , p. $1239-1247$.

[11] MOLINDER, J. I. A tutorial introduction to very long baseline interferometry (VLBI) using bandwidth synthesis. DSN Progress Report 42-46. California (USA). Jet Propulsion Laboratory, 1978, p. $16-28$.

[12] GORHAM, P.W. Designing optimal bandwidth synthesis arrays for VLBI. TMO Progress Report 42-133. California (USA). Jet Propulsion Laboratory, 1998, p. 1-27.

[13] BAGRI, D.S. A proposed frequency synthesis approach to accurately measure the angular position. IPN Progress Report 42163. California (USA). Jet Propulsion Laboratory, 2005, p. 1-7.

[14] KONDO, T. Bandwidth synthesis of VLBI data with clock-offsets different every channel. In Japan Geoscience Union Meeting. Chiba (Japan), 2007, p. 1-3.

[15] XIANG-WEI ZHU, YUAN-LING LI, SHAO-WEI YONG, ET AL. A novel definition and measurement method of group delay and its application. IEEE Transactions on Instrumentation and Measurement, 2009, vol. 58, no. 1, p. 229-233. DOI: 10.1109/TIM.2008.927197

[16] Alan R. Whitney. VLBI digital-backend intercomparison testing (Draft). MIT Haystack Observatory. Westford (USA), 2009, p. 1-5.

[17] WhitNeY, AL., BEAUDOIN, CH., CAPPALlO, R., ET al. VLBI digital-backend intercomparison test report. MIT Haystack Observatory. Westford (USA), 2012, p. 1-18.

[18] JIANG KUN, HOU XIAO-MIN, XU KE, ET AL. High efficiency parallel extraction of multi-tone PCAL signals. Journal of Spacecraft TT\&C Technology, 2012, vol. 31, no. 6, p. 32-36.

[19] JIANG KUN, JIAO YIWEN, HOU XIAOMIN, ET AL. Design and implementation of the deep space interferometry DBBC subsystem. In International Conference on Signal Processing ICSP2012. Beijing (China), 2012, p. 448-451. DOI: 10.1109/ICoSP.2012.6491521

\section{About the Authors ...}

Kun JIANG was born in Luoyang, China, in 1984. He received the Ph.D. degree in Communication and Information Systems from the Academy of Equipment, Beijing, China, in 2013. He is now working in the Beijing 
Inst. of Tracking and Telecommunication Technology. His research interests include global navigation satellite system, TT\&C, digital baseband conversion, and VLBI.

Pingbo YAN was born in Changzhou, China, in 1961. He received the M.S. degree from the Nanjing University of Science and Technology, China, in 1997. He is the senior engineer and M.S. supervisor of the Academy of Equipment. His research interests include signal processing and experiment design.

Yuanqin WANG was born in Mudanjiang, China, in 1963. He received the Ph.D. from Harbin Inst. of Technology, China, in 2011. He is a professor and Ph.D. supervisor of the Academy of Equipment. His research interests include TT\&C, high speed digital signal processing.

Yiwen JIAO was born in Xianghe, China, in 1985. He is currently working toward the Ph.D. degree in Communication and Information Systems with the TT\&C Research Center, Dept. of Optical and Electrical Equipment. His research interests include $\mathrm{TT} \& \mathrm{C}$, ultra wideband signal processing, VLBI.

Xin LIAN was born in Changchun, China, in 1987. He is currently working toward the Ph.D. degree in Communication and Information Systems with the TT\&C Research Center, Dept. of Optical and Electrical Equipment. His research interests include $\mathrm{TT} \& \mathrm{C}$, ultra wideband signal processing, VLBI.

Ke XU was born in Xianyang, China, in 1988. He received the M.S. degree in Information and Communication Engineering from the Academy of Equipment, Beijing, China, in 2012. His research interests include TT\&C and VLBI signal processing. 